\title{
Spectrum of the Vortex Bound States of the Dirac and Schrodinger Hamiltonian in the presence of Superconducting Gaps
}

\author{
Chi-Ho Cheng* \\ Department of Physics, National Changhua University of Education, Taiwan
}

(Dated: October 10, 2018)

\begin{abstract}
We investigate the vortex bound states both Schrodinger and Dirac Hamiltonian with the s-wave superconducting pairing gap by solving the mean-field Bogoliubov-de-Gennes equations. The exact vortex bound states spectrum is numerically determined by the integration method, and also accompanied by the quasi-classical analysis. It is found that the bound state energies is proportional to the vortex angular momentum when the chemical potential is large enough. By applying the external magnetic field, the vortex bound state energies of the Dirac Hamiltonian are almost unchanged; whereas the energy shift of the Schrodinger Hamiltonian is proportional to the magnetic field. These qualitative differences may serve as an indirect evidence of the existence of Majorana fermions in which the zero mode exists in the case of the Dirac Hamiltonian only.
\end{abstract}

PACS numbers: 71.27.+a, 74.45.+c, 71.10.Pm

\section{INTRODUCTION}

The zero mode (Majorana fermion excitation) attracts lots of investigation due to its non-Abelian statistics [13] and possible application to the fault tolerant quantum processing [4, 5]. Possible candidates to support Majorana fermions are $p+i p$ superconductors [2, 6, 7], $p+i p$ superfluids in cold atoms [8 10]. With the proximity effect between the $s$-wave superconductor and the strong topological insulator surface 11 14, chiral Majorana fermions could be created as edge states 15]. Besides that, Majorana fermions could also be realized in semiconductor with spin-orbit coupling [16 18].

To verify the existence of Majorana fermion, Law et al. proposed the tunneling experiments to probe the chiral Majorana fermion at the interface between a superconductor and the surface of a topological insulator, in which the Majorana fermions induce resonant Andreev reflection [19].

In this paper, we investigate and compare the vortex bound states of both the Dirac and Schrodinger Hamiltonian with the s-wave superconducting gap by solving the mean-field Bogoliubov-de-Gennes equations. The bound states are numerically solved by the integration method as well as the quasi-classical analysis. Their differences could be served as an indirect verification of the existence of the Majorana fermion at the surface of the topological insulator with an induced superconducting gap due to the proximity effect.

\section{FORMULATION OF VORTEX BOUND STATES}

Our formulation is based on the mean-field Bogoliubov-de-Gennes (BdG) equations for the quasiparticle. In terms of Nambu indices, the mean-field
Hamiltonian becomes

$$
H=\frac{1}{2} \int d^{2} x\left(\begin{array}{ll}
\Psi^{\dagger} & \Phi^{\dagger}
\end{array}\right)\left(\begin{array}{cc}
H_{0}^{(D, S)} & i \sigma_{y} \Delta(\vec{r}) \\
-i \sigma_{y} \Delta^{*}(\vec{r}) & -H_{0}^{(D, S) *}
\end{array}\right)\left(\begin{array}{c}
\Psi \\
\Phi
\end{array}\right)
$$

where $\Psi=\left(c_{\uparrow}, c_{\downarrow}\right)^{T}$ and $\Phi=\left(c_{\uparrow}^{\dagger}, c_{\downarrow}^{\dagger}\right)^{T} . H_{0}^{(D, S)}$ in this paper could be the Dirac Hamiltonian, $H_{0}^{(D)}=v_{\mathrm{F}} \vec{p} \cdot \vec{\sigma}-$ $\mu-h \sigma_{z}$, and the Schrodinger Hamiltonian, $H_{0}^{(S)}=\frac{p^{2}}{2 m}-$ $\mu-h \sigma_{z} \cdot v_{\mathrm{F}}$ denotes the Fermi velocity, $\mu$ is the chemical potential, $h$ represents the Zeeman coupling due to the external magnetic field acting along the $z$-direction, and $\Delta(\vec{r})$ is the superconducting gap function.

The quasi-particle states of the above Hamiltonian in Eq.(11) are given by

$$
\left(\begin{array}{cc}
H_{0}^{(D, S)} & i \sigma_{y} \Delta(\vec{r}) \\
-i \sigma_{y} \Delta^{*}(\vec{r}) & -H_{0}^{(D, S) *}
\end{array}\right)\left(\begin{array}{c}
\mathbf{u}_{n}(\vec{r}) \\
\mathbf{v}_{n}(\vec{r})
\end{array}\right)=E_{n}\left(\begin{array}{c}
\mathbf{u}_{n}(\vec{r}) \\
\mathbf{v}_{n}(\vec{r})
\end{array}\right)(2)
$$

Considering an isolated vortex carrying one flux quantum, i.e. $\Delta(\vec{r})=\Delta(r) \mathrm{e}^{i \phi}$, with the eigenvectors of the form, $\mathbf{u}_{n}(\vec{r})=\left(u_{\uparrow, n}(r) \mathrm{e}^{i n \phi}, u_{\downarrow, n}(r) \mathrm{e}^{i(n+1) \phi}\right)$ and $\mathbf{v}_{n}(\vec{r})=$ $\left(v_{\uparrow, n}(r) \mathrm{e}^{i n \phi}, v_{\downarrow, n}(r) \mathrm{e}^{i(n-1) \phi}\right)$. The eigenproblem for the Dirac Hamiltonian in radial coordinate becomes

$$
\left(\begin{array}{cc}
K_{n}^{(D)} & i \sigma_{y} \Delta(r) \\
-i \sigma_{y} \Delta(r) & -K_{n-1}^{(D) \dagger}
\end{array}\right)\left(\begin{array}{c}
\widetilde{u}_{n}(r) \\
\widetilde{v}_{n}(r)
\end{array}\right)=E_{n}\left(\begin{array}{c}
\widetilde{u}_{n}(r) \\
\widetilde{v}_{n}(r)
\end{array}\right)
$$

where

$$
K_{n}^{(D)}=\left(\begin{array}{cc}
-\mu-h & -i \hbar v_{\mathrm{F}}\left(\frac{\partial}{\partial r}+\frac{n+1}{r}\right) \\
-i \hbar v_{\mathrm{F}}\left(\frac{\partial}{\partial r}-\frac{n}{r}\right) & -\mu+h
\end{array}\right)
$$

and $\quad \widetilde{u}_{n}(r)=\left(u_{\uparrow, n}(r), u_{\downarrow, n}(r)\right)^{T}, \quad \widetilde{v}_{n}(r)=$ $\left(v_{\uparrow, n}(r), v_{\downarrow, n}(r)\right)^{T}$.

For the Schrodinger Hamiltonian, the eigenproblem can be further block-diagonalized into

$$
\left(K_{n}^{(S)}+\sigma_{x} \Delta(r)-h\right)\left(\begin{array}{c}
u_{\uparrow, n}(r) \\
v_{\downarrow, n}(r)
\end{array}\right)=E_{n}\left(\begin{array}{c}
u_{\uparrow, n}(r) \\
v_{\downarrow, n}(r)
\end{array}\right)
$$




$$
\left(K_{n+1}^{(S)}-\sigma_{x} \Delta(r)+h\right)\left(\begin{array}{c}
u_{\downarrow, n}(r) \\
v_{\uparrow, n}(r)
\end{array}\right)=E_{n}\left(\begin{array}{c}
u_{\downarrow, n}(r) \\
v_{\uparrow, n}(r)
\end{array}\right)
$$

with
(6)

$$
K_{n}^{(S)}=\left(\begin{array}{cc}
-\frac{\hbar^{2}}{2 m}\left(\frac{\partial^{2}}{\partial r^{2}}+\frac{1}{r} \frac{\partial}{\partial r}-\frac{n^{2}}{r^{2}}\right)-\mu & 0 \\
0 & \frac{\hbar^{2}}{2 m}\left(\frac{\partial^{2}}{\partial r^{2}}+\frac{1}{r} \frac{\partial}{\partial r}-\frac{(n-1)^{2}}{r^{2}}\right)+\mu
\end{array}\right)
$$

Eqs.(5) and (6) are not independent to each other. In fact, their complete sets of eigenvectors are the same.

The size of the vortex core is characterized by the coherent length $\xi$. For the Dirac Hamiltonian, it should be of the order of $\hbar v_{\mathrm{F}} / \Delta_{0}$, the unique length scale of the system, where $\Delta_{0}$ is the superconducting gap far away from the vortex core. However, for the Schrodinger Hamiltonian, there are two length scales $k_{\mathrm{F}}^{-1}$ and $\hbar v_{\mathrm{F}} / \Delta_{0}$. $\xi$ is of the order of $\hbar v_{\mathrm{F}} / \Delta_{0}$ when $\mu / \Delta_{0} \gg 1$. As $\mu / \Delta_{0}$ becomes smaller, $\xi$ is going to shrink to another length scale, $k_{\mathrm{F}}^{-1}$ [24 26]. When $\mu / \Delta_{0}$ turns to be negative, a new length scale due to the bosonic molecules emerges.

We adopt $\xi=\hbar v_{\mathrm{F}} / \Delta_{0}$ for the Dirac Hamiltonian, and $\xi=\hbar c / \Delta_{0}$ for the Schrodinger Hamiltonian in which $c$ is a constant independent of the chemical potential $\mu$. Further we assume the form of the gap amplitude

$$
\Delta(r)=\Delta_{0} \tanh \left(\frac{r}{\xi}\right)
$$

The BdG equations, Eqs.(3) and (5), are solved by the integration method. Imposing that the wavefunction aymptotically approaches to zero far away from the vortex core, the eigenenergies can be obtained by integrating the differential equation from the infinity to the vortex center. The advantage of the integration method is that we can solve for the numerically exact (that is, system size in the thermodynamical limit) bound state energies once the form of the gap amplitude as in Eq.(8) is given.

The chemical potential $\mu$ in the BdG equations for the Schrodinger Hamiltonian can be positive or negative, arbitrary small or large. The condition of the quasi-classical approximation, $k_{\mathrm{F}} \xi \gg 1$ (equivalent to $\mu / \Delta_{0} \gg 1$ ), may not be valid in the whole range of the chemical potential. Eq. (5) is solved directly by the integration method. We do not assume the Andreev (quasiclassical) approximation [23 25].

\section{NUMERICAL RESULTS}

We first consider the case without external magnetic field. The vortex bound state energies of the Dirac Hamiltonian are solved for different chemical potential.
The vortex bound state energies of the Dirac Hamiltonian as function of chemical potential $\mu$ is shown in Fig.1. We do not show the negative $n$ because $E_{-n}=$ $-E_{n}$ which is the consequence of time-reversal symmetry. That is, $E \rightarrow-E$ when $n \rightarrow-n$ (reverse the angular momentum). Besides that, $E \rightarrow-E$ when $\mu \rightarrow-\mu$, which is the particle-hole symmetry particularly for the Dirac Hamiltonian.

For $n=0$, the zero mode corresponds to the Majorana fermions. For non-zero angular momentum, $n>0,|\mu| \gg$ $\Delta_{0}$, we found the mid-gap state near zero energy. As $\mu$ approaches to zero, the mid-gap state gradually merges to the continuum of energy $E=\Delta_{0}$. Especially, near zero chemical potential, $\mu \simeq 0$, all the vortex bound states except the Majorana fermions state, are located near the edge.

We also plot the bound state energy as a function of the angular momentum quantum number $n$, as shown in Fig 2. There is a simple linear relationship $E \propto n$ for small $|n|$ (up to $n=10$ ) when $\mu \gg \Delta_{0}$. This linear relationship could be explained by quasi-classical analysis in the next section. As $\mu \simeq 0$, the bound state energy except the zero mode merges into the continuum $\Delta_{0}$. The prediction of the bound state energies by linearizing the vortex amplitude [20, 21] is proportional to $\sqrt{N}(N$ is an integer quantum number), which is inconsistent with our calculation.

We also solve for the bound state energies of the Schrodinger Hamiltonian for comparison. Fig 3 shows the bound state energies as a function of $\mu$, with $m c^{2} / \Delta_{0}=10$. Due to the time-reversal symmetry, $E_{-n}=-E_{n-1}$. Unlike the Dirac case, no particle-hole symmetry remains. For highly positive chemical potential $\mu \gg \Delta_{0}$, the qualitative behavior is the same as the Dirac case except that the zero mode is missing. A simple linear relationship $E \propto n$ is also recovered, as shown in Fig 4. The negative chemical potential, $\mu<0$, can be realized in superfluid Fermi gases near the BEC to BCS crossover 26]. For $\mu<0$, the continuum of the excitation becomes $\sqrt{\mu^{2}+\Delta_{0}^{2}}$. The mid-gap state merges into the continuum when the chemical potential is deeply negative, $\mu \ll-\Delta_{0}$. The qualitative behavior of the midgap states remains the same for different $c$, which is also shown in Figs 5 and 6 with $m c^{2} / \Delta_{0}=1$. 
Under the external magnetic field perpendicular to the surface, the vortex bound states of the Dirac and Schrodinger Hamiltonian behave very differently.

For the Dirac Hamiltonian, the continuum threshold of bound state energy is $\Delta_{0} \sqrt{1-h^{2} / \Delta_{0}^{2}}$ when $0<h<\mu / \sqrt{1+\Delta_{0}^{2} / \mu^{2}}$, and $\sqrt{\mu^{2}+\Delta_{0}^{2}}-h$ when $\mu / \sqrt{1+\Delta_{0}^{2} / \mu^{2}}<h<\sqrt{\mu^{2}+\Delta_{0}^{2}}$, and the energy becomes fully continuum when $h>\sqrt{\mu^{2}+\Delta_{0}^{2}}$.

In the limit of $|\mu| \gg \Delta_{0}$, as illustrated in Fig 7 for $\mu / \Delta_{0}=10$, the vortex bound states of different angular momenta are equally spaced. As the external magnetic field increases, the vortex bound states merge into the continuum. Notice that all the states are insensitive under the external magnetic field.

As $|\mu|$ decreases, the vortex bound states of finite angular momentum moves towards the continuum, as illustrated in Fig 8 for $\mu / \Delta_{0}=1$. When $|\mu| \ll \Delta_{0}$, the zero mode exists until it meets the continuum when $h>\sqrt{\mu^{2}+\Delta_{0}^{2}}$. The remaining bound states are located near the edge of continuum. The case that $\mu / \Delta_{0}=0$ is shown in Fig 9 ,

For the Schrodinger Hamiltonian, it is easy to see from Eqs.(5)-(6) that the vortex bound state energy shift due to the external magnetic field is $\pm h$. This is the fundamental difference from the Dirac Hamiltonian.

\section{QUASI-CLASSICAL ANALYSIS}

\section{A. Dirac Hamiltonian}

From Eq.(2), the eigenvector of the zero energy state is given by

$$
\left(\begin{array}{cc}
v_{\mathrm{F}} \vec{p} \cdot \vec{\sigma}-\mu-h \sigma_{z} & i \sigma_{y} \Delta \\
-i \sigma_{y} \Delta^{*} & -v_{\mathrm{F}} \vec{p} \cdot \vec{\sigma}+\mu+h \sigma_{z}
\end{array}\right)\left(\begin{array}{l}
\mathbf{u} \\
\mathbf{v}
\end{array}\right)=0
$$

Under the condition of the quasi-classical approximation, i.e., $k_{\mathrm{F}} \xi \gg 1$, the wavelength of the quasi-particles is much smaller than the coherent length of the vortex, their trajectories are almost straight lines. Following the argument by Volovik [27], the zero energy state corresponds to the trajectory that crosses the center of the vortex, i.e., zero impact parameter, $b=0$. The superconducting gap function

$$
\Delta(s)=\Delta(|s|) \operatorname{sgn}(s)
$$

where $s$ parameterizes the trajectory across the vortex center.

For $|h|<|\mu|$, by directly solving Eqs.(91)-(10), we have the zero energy state

$$
\left(\begin{array}{c}
\mathbf{u} \\
\mathbf{v}
\end{array}\right)=\frac{\mathrm{e}^{i q s} I \otimes \mathrm{e}^{\frac{i}{2} \sigma_{z} \theta}}{2 \sqrt{\mu(\mu-h)}}\left(\begin{array}{c}
(\mu-h) \\
\sqrt{\mu^{2}-h^{2}} \\
i(\mu-h) \\
-i \sqrt{\mu^{2}-h^{2}}
\end{array}\right) \psi(s)
$$

where the plane wave momentum $\vec{q}=\frac{\sqrt{\mu^{2}-h^{2}}}{\hbar v_{\mathrm{F}}}\left(\hat{\mathrm{e}}_{x} \cos \theta+\right.$ $\hat{\mathrm{e}}_{y} \sin \theta$ ), and

$$
\psi(s)=\exp \left(-\frac{1}{\hbar v_{\mathrm{F}}} \int^{s} d s^{\prime} \Delta\left(\left|s^{\prime}\right|\right) \operatorname{sgn}\left(s^{\prime}\right)\right)
$$

The non-zero angular momentum vortex bound state near the zero energy state can be estimated by small impact parameter $b, \Delta\left(r=\sqrt{s^{2}+b^{2}}\right) \mathrm{e}^{i \phi} \simeq \Delta(|s|) \operatorname{sgn}(\mathrm{s})+$ $i \Delta(|s|) b / s$. Hence we have the perturbation

$$
H^{\prime}=\frac{i \Delta(|s|) b}{s}\left(\begin{array}{cc}
0 & i \sigma_{y} \\
i \sigma_{y} & 0
\end{array}\right)
$$

The bound state energies up to first order correction becomes

$$
E=\left\langle H^{\prime}\right\rangle=\operatorname{sgn}(\mu) b \frac{\int_{-\infty}^{\infty} d s \frac{\Delta(|s|)}{s}|\psi(s)|^{2}}{\int_{-\infty}^{\infty} d s|\psi(s)|^{2}}
$$

and the angular momentum $L_{z}=-p b=-b|\mu| / v_{\mathrm{F}}$ which is $n \hbar$ by the semi-classical quantization condition,

$$
E=-n \hbar \omega_{0}
$$

with

$$
\omega_{0}=\frac{v_{\mathrm{F}}}{\mu} \frac{\int_{-\infty}^{\infty} d s \frac{\Delta(|s|)}{s}|\psi(s)|^{2}}{\int_{-\infty}^{\infty} d s|\psi(s)|^{2}}
$$

Notice that $\omega_{0}$ has no $h$ dependence.

On the other hand, when $|h|>|\mu|$, the zero energy state is a decaying solution,

$$
\left(\begin{array}{c}
\mathbf{u} \\
\mathbf{v}
\end{array}\right)=\frac{\mathrm{e}^{-\kappa s} I \otimes \mathrm{e}^{\frac{i}{2} \sigma_{z} \theta}}{2 \sqrt{\mu(\mu-h)}}\left(\begin{array}{c}
h-\mu \\
-i \sqrt{h^{2}-\mu^{2}} \\
i(h-\mu) \\
\sqrt{h^{2}-\mu^{2}}
\end{array}\right) \psi(s)
$$

where $\kappa=\frac{\sqrt{h^{2}-\mu^{2}}}{\hbar v_{\mathrm{F}}}$. The correction $\left\langle H^{\prime}\right\rangle=0$. It is consistent with all finite angular momentum bound states merging to zero bound state at $h=\mu$.

\section{B. Schrodinger Hamiltonian}

For the case of the Schrodinger Hamiltonian with superconducting gap, the eigenproblem for the quasiparticles is

$$
\left(\left(\frac{p^{2}}{2 m}-\mu\right) \sigma_{z}+\left(\begin{array}{cc}
0 & \Delta \\
\Delta^{*} & 0
\end{array}\right)-h\right)\left(\begin{array}{l}
u_{\uparrow} \\
v_{\downarrow}
\end{array}\right)=E\left(\begin{array}{l}
u_{\uparrow} \\
v_{\downarrow}
\end{array}\right)
$$

In the quasi-classical approach, after the transformation $\left(u_{\uparrow}, v_{\downarrow}\right) \rightarrow \mathrm{e}^{i \vec{q} \cdot \vec{r}}\left(u_{\uparrow}, v_{\downarrow}\right)$, the eigenproblem becomes 


$$
\begin{array}{r}
\left(\left(\vec{v}_{\mathrm{F}} \cdot \vec{p}-\frac{\hbar^{2}}{2 m r^{2}} \frac{\partial^{2}}{\partial \phi^{2}}\right) \sigma_{z}+\left(\begin{array}{cc}
0 & \Delta \\
\Delta^{*} & 0
\end{array}\right)-h\right)\left(\begin{array}{c}
u_{\uparrow} \\
v_{\downarrow}
\end{array}\right)=E\left(\begin{array}{c}
u_{\uparrow} \\
v_{\downarrow}
\end{array}\right) \\
\left.\left(\left(\vec{v}_{\mathrm{F}} \cdot \vec{p}\right) \sigma_{z}+\sigma_{x} \Delta(s)\right)\left(\begin{array}{c}
u_{\uparrow} \\
v_{\downarrow}
\end{array}\right)=\left(E+h+\frac{\hbar^{2} \sigma_{z}}{2 m r^{2}} \frac{\partial^{2}}{\partial \phi^{2}}-i \sigma_{y} \frac{i \Delta(|s|) b}{s}\right)\right)\left(\begin{array}{c}
u_{\uparrow} \\
v_{\downarrow}
\end{array}\right)
\end{array}
$$

where $\vec{q}=\frac{\sqrt{2 m \mu}}{\hbar}\left(\hat{\mathrm{e}}_{x} \cos \theta+\hat{\mathrm{e}}_{y} \sin \theta\right)$, and $\vec{v}_{\mathrm{F}}=\hbar \vec{q} / m$, for $\mu>0$.

We solve the left hand side, and treat the right hand side to the first order perturbation [22]. The bound state energy is

$$
E=-\left(n-\frac{1}{2}\right) \hbar \omega_{0}-h
$$

with $\omega_{0}$ defined in Eq.(16). $n$ is an integer. The half integer $\frac{1}{2}$ is due to the contribution of the term $-\frac{\hbar^{2} \sigma_{z}}{2 m r^{2}} \frac{\partial^{2}}{\partial \phi^{2}}$ which is absent in the Dirac Hamiltonian. Because of the extra half integer, there is no zero mode in the vortex bound state of the Schrodinger Hamiltonian.

The bound state energy for $\left(u_{\downarrow}, v_{\uparrow}\right)$ can be obtained from the particle-hole transformation, which gives

$$
E=\left(n-\frac{1}{2}\right) \hbar \omega_{0}+h
$$

\section{CONCLUSION}

In conclusion, we solve numerically and perform the quasi-classical analysis for the vortex bound states of both the Dirac and Schrodinger Hamiltonians in the presence of superconducting gap. It was found that the bound state energies follows the linear relationship with the angular momentum for both cases when the chemical potential is highly positive, $\mu \gg \Delta_{0}$. If the chemical potential is highly negative, $\mu \ll-\Delta_{0}$, the linear relationship holds for the Dirac Hamiltonian due to the particle-hole symmetry; whereas the bound states for the Schrodinger Hamiltonian merge into the continuum. After applying the external magnetic field perpendicular to the interface, the bound states energies for the Dirac Hamiltonian are insensitive; whereas there is a Zeeman shift for the Schrodinger Hamiltonian. The bound states should be determined by the local density of states on the surface from the scanning tunneling microscopy experiments. The bound state energy differences can be determined by the absorption of circular polarized light. The qualitative differences between the Schrodinger and Dirac Hamiltonian could serve as an indirect evidence of the existence of Majorana fermions.

\section{ACKNOWLEDGEMENT}

I would thank very much Sungkit Yip for his help to my understanding of the subject matter, and further discussion. The work was supported by the National Science Council of the Republic of China.

* Electronic address: phcch@cc.ncue.edu.tw

[1] G. Moore and N. Read, Nucl. Phys. B 360, 362 (1991).

[2] N. Read and D. Green, Phys. Rev. B 61, 10267 (2000).

[3] D. A. Ivanov, Phys. Rev. Lett. 86, 268 (2001).

[4] A. Kitaev, Ann. Phys. 303, 2 (2003).

[5] C. Nayak, S. H. Simon, A. Stern, M. Freedman, and S. Das Sarma, Rev. Mod. Phys. 80, 1083 (2008).

[6] A. Stern, F. von Oppen, and E. Mariani, Phys. Rev. B 70, 205338 (2004).

[7] M. Stone and S. B. Chung, Phys. Rev. B 73, 014505 (2006).

[8] V. Gurarie, L. Radzihovsky, and A. V. Andreev, Phys. Rev. Lett. 94, 230403 (2005).

[9] C. H. Cheng and S. K. Yip, Phys. Rev. Lett. 95, 070404 (2005).

[10] S. Tewari, S Das Sarma, C. Nayak, C. Zhang, and P. Zoller, Phys. Rev. Lett. 98, 010506 (2007).

[11] L. Fu, C. L. Kane, and E. J. Mele, Phys. Rev. Lett. 98, 106803 (2007).

[12] J. E. Moore and L. Balents, Phys. Rev. B 75, 121306(R) (2007).

[13] L. Fu and C. L. Kane, Phys. Rev. B 76, 045302 (2007).

[14] R. Roy, Phys. Rev. B 79, 195322 (2009).

[15] L. Fu and C. L. Kane, Phys. Rev. Lett. 100, 096407 (2008).

[16] J. D. Sau, R. M. Lutchyn, S. Tewari, and S. Das Sarma, Phys. Rev. Lett. 104, 040502 (2010).

[17] J. Alicea, Phys. Rev. B 81, 125318 (2010).

[18] A. C. Potter and P. A. Lee, Phys. Rev. Lett. 105, 227003 (2010).

[19] K. T. Law, P. A. Lee, and T. K. Ng, Phys. Rev. Lett. 103, 237001 (2009).

[20] C. K. Lu and I. F. Herbut, Phys. Rev. B 82, 144505 (2010).

[21] I. F. Herbut and C. K. Lu, Phys. Rev. B 83, 125412 (2011).

[22] P. G. de Gennes, Superconductivity of Metals and Alloys, Addison-Wesley (1989).

[23] W. L. Clinton, Phys. Rev. B 46, 5742 (1992).

[24] L. Kramer and W. Pesch, Z. Phys. 269, 59 (1974).

[25] F. Gygi and M. Schluter, Phys. Rev. B 41, 822 (1990).

[26] R. Sensarma, M. Randeria, and T.-L. Ho, Phys. Rev. 
Lett. 96, 090403 (2006).

[27] G. E. Volovik, JETP Letters 70, 609 (1999).

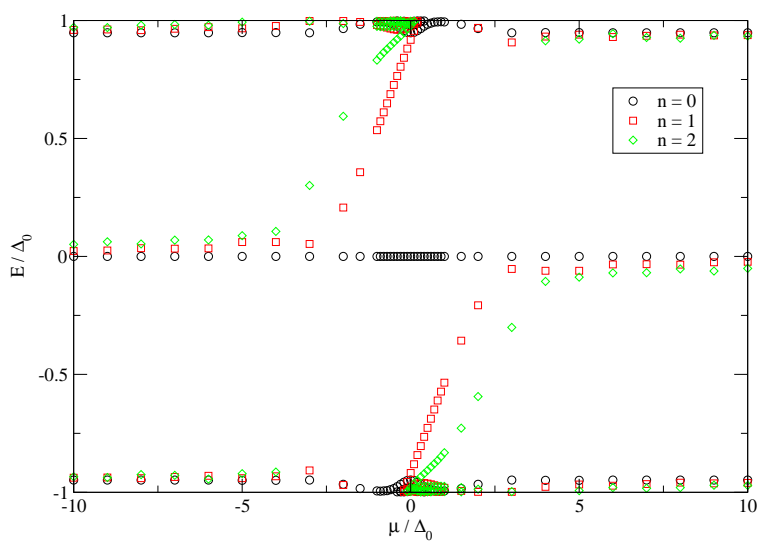

FIG. 1: Vortex bound state energies of the Dirac Hamiltonian as a function of chemical potential $\mu$ in the absence of external magnetic field, $h=0$, for different angular momentum label by quantum number $n$. $E_{-n}=-E_{n}$.

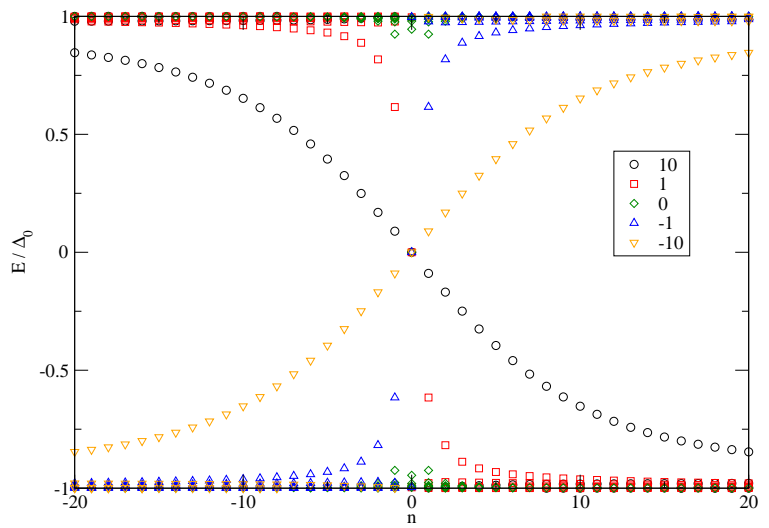

FIG. 2: Vortex bound state energies of the Dirac Hamiltonian as a function of angular momentum quantum numbers $n$ in the absence of external magnetic field, $h=0$, for $\mu / \Delta_{0}=$ $10,1,0,-1,-10$. 


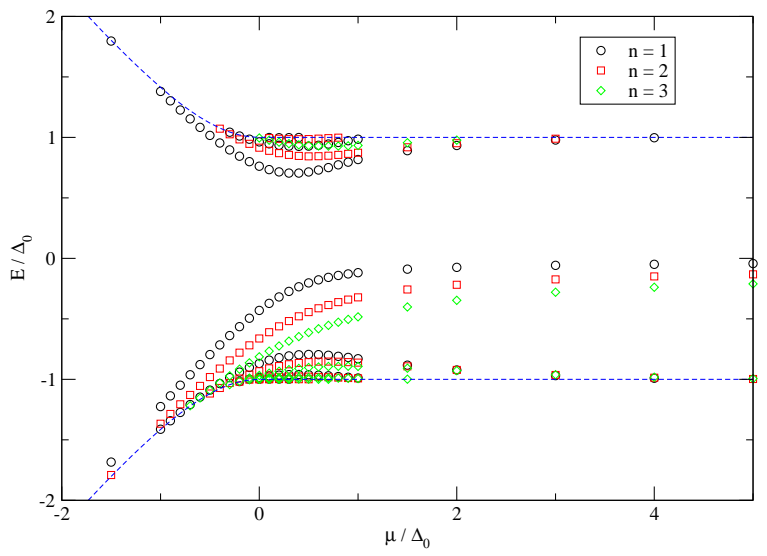

FIG. 3: Vortex bound state energies of the Schrodinger Hamiltonian as a function of chemical potential $\mu$ in the absence of external magnetic field, $h=0$, for different angular momentum label by quantum number $n . m c^{2} / \Delta_{0}=10$. $E_{-n}=-E_{n-1}$. The dashed lines represent the continuum threshold.

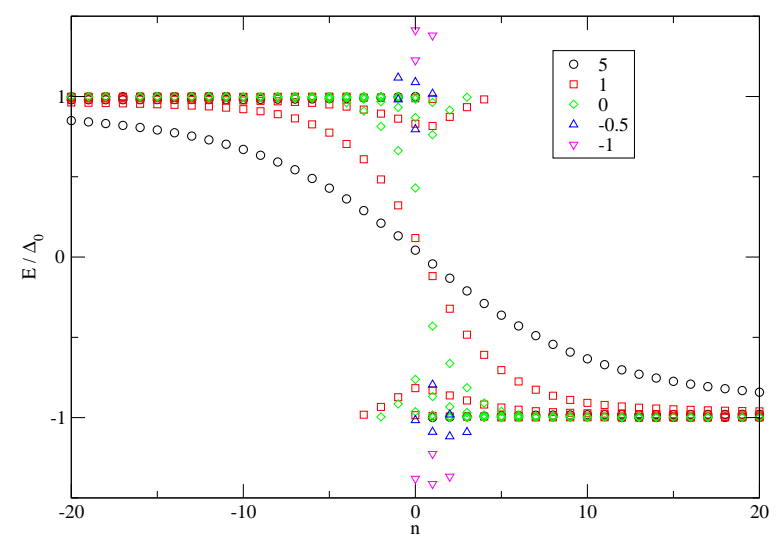

FIG. 4: Vortex bound state energies of the Schrodinger Hamiltonian as a function of angular momentum quantum numbers $n$ in the absence of external magnetic field, $h=0$, for $\mu / \Delta_{0}=5,1,0,-0.5,-1$ when $m c^{2} / \Delta_{0}=10$. 


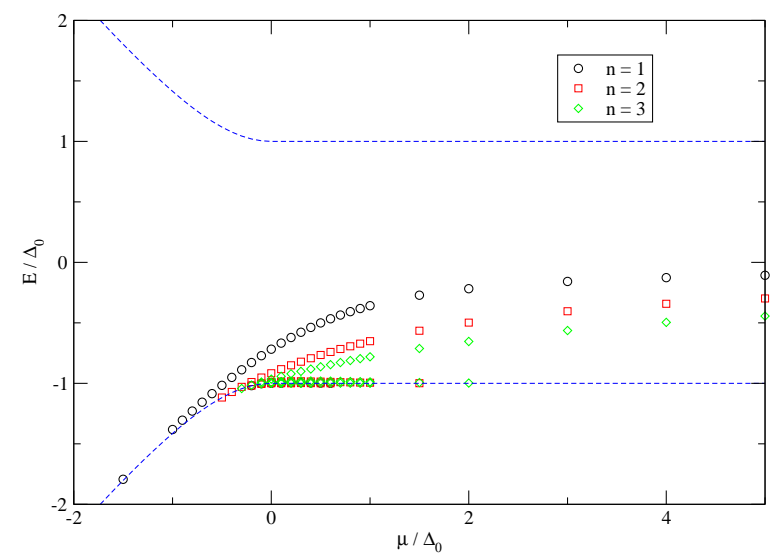

FIG. 5: Vortex bound state energies of the Schrodinger Hamiltonian as a function of chemical potential $\mu$ in the absence of external magnetic field, $h=0$, for different angular momentum label by quantum number $n . m c^{2} / \Delta_{0}=1$. $E_{-n}=-E_{n-1}$. The dashed lines represent the continuum threshold.

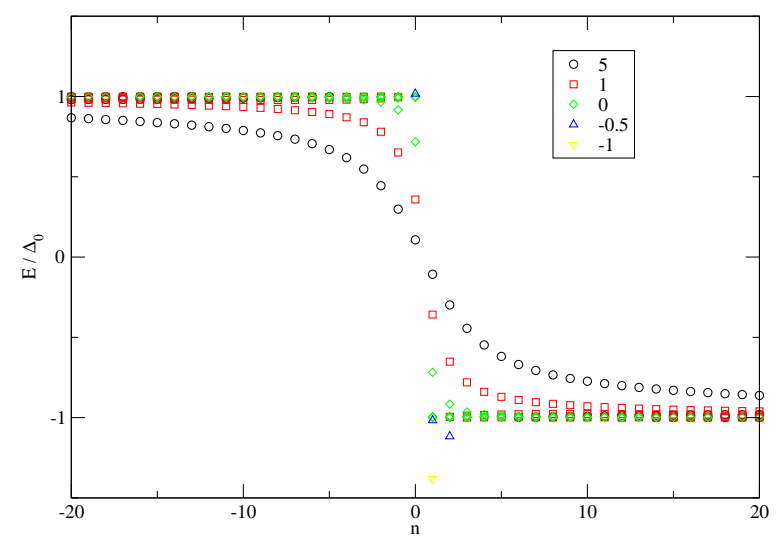

FIG. 6: Vortex bound state energies of the Schrodinger Hamiltonian as a function of angular momentum quantum numbers $n$ in the absence of external magnetic field, $h=0$, for $\mu / \Delta_{0}=5,1,0,-0.5,-1$ when $m c^{2} / \Delta_{0}=1$. 


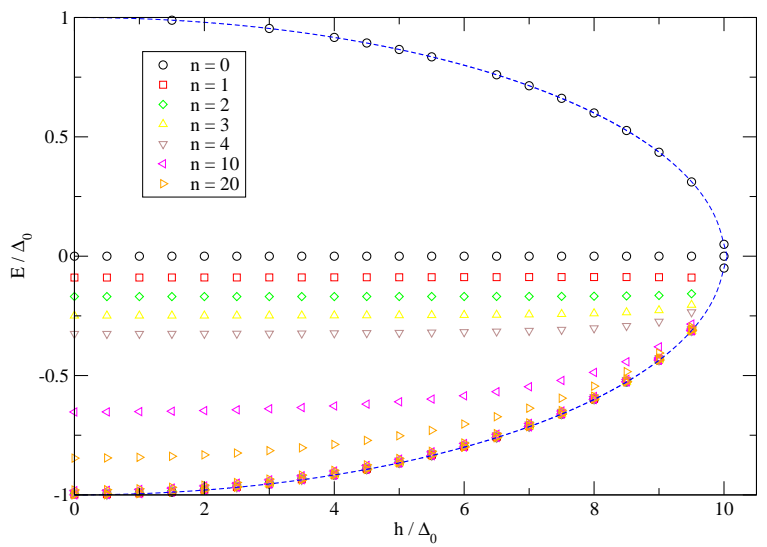

FIG. 7: Vortex bound state energies of the Dirac Hamiltonian as a function of external magnetic field $h$ at $\mu / \Delta_{0}=10$, for different $n$. The dashed lines represent the continuum threshold.

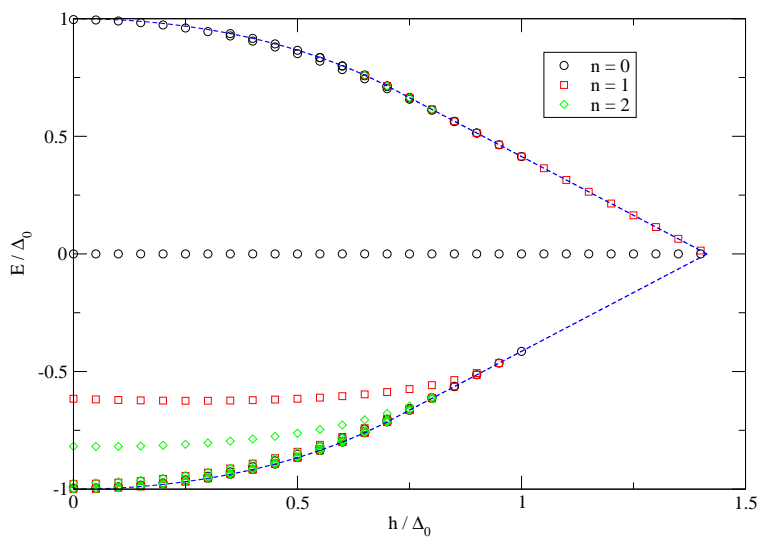

FIG. 8: Vortex bound state energies of the Dirac Hamiltonian as a function of external magnetic field $h$ at $\mu / \Delta_{0}=1$, for different $n$. The dashed lines represent the continuum threshold. 


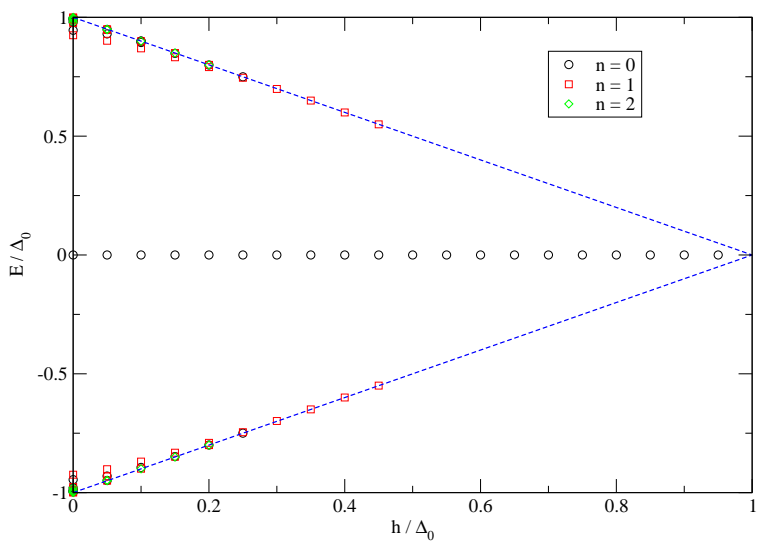

FIG. 9: Vortex bound state energies of the Dirac Hamiltonian as a function of external magnetic field $h$ at $\mu / \Delta_{0}=0$, for different $n$. The dashed lines represent the continuum threshold. 